\title{
Unusual Temperature-Induced Swelling of Ionizable Poly $(N-$ isopropylacrylamide)-Based Microgels: Experimental and Theoretical Insights into its Molecular Origin
}

\author{
Juan M. Giussi, ${ }^{1}$ Manuel I. Velasco, ${ }^{2}$ Gabriel S. Longo, ${ }^{1}{ }^{\text {Rodolfo H. }}$ Acosta ${ }^{2}$ and Omar \\ Azzaroni $^{{ }^{*}}$ \\ ${ }^{1}$ Instituto de Investigaciones Fisiccoquímicas Teóricas y Aplicadas (INIFTA) - Departamento de Química - \\ Facultad de Ciencias Exactas - Universidad Nacional de La Plata - CONICET - (1900) La Plata - \\ Argentina \\ ${ }^{2}$ FaMAF-Universidad Nacional de Córdoba and IFEG-CONICET, X5016LAE Córdoba, Argentina
}

\begin{abstract}
In the traditional view of temperature-driven volume phase transitions in PNIPAM-based microgel solutions, a monotonic and sharp decrease in particle size occurs upon heating the solution above the volume phase transition temperature (VPTT). However, at sufficiently high microgel concentrations and low salt conditions, our dynamic light scattering experiments reveal an unexpected non-monotonic evolution of particle size when increasing the solution temperature. These findings show that poly(N-isopropylacrylamide-co-methacrylic acid) (P(NIPAM-co-MAA)) microgels swell upon heating the solution in the temperature range where NIPAM is water-soluble (i.e., below the VPPT). Further heating the microgel solution leads to microgel collapse as typically observed at temperatures above the VPTT. This novel behavior depends on particle and salt concentration. We have observed the expected monotonic temperature-response of P(NIPAm-coMAA) microgel solutions at low particle density and high salt concentration. To gain insights into the molecular origin of the unusual behavior of these microgel solutions, we have combined nuclear magnetic resonance studies and molecular-level theoretical calculations of the system. A delicate balance between inter-particle steric compressions and intra-microgel physical interactions and chemical equilibria determines the size of these microgels. Both steric compression, due to finite density, and hydrogen bond formation in the interior of the microgels favor a more compact particle. On the contrary, at the $\mathrm{pH}$ of the experiments the acid-base equilibrium constraints the polymer charge to increase, which favors particle swelling due to intra-microgel electrostatic repulsions. This interplay between physical interactions and chemical equilibria occurring at the nanometer length-scale determines the unusual thermal-induced swelling of P(NIPAM-co-MAA) microgels.
\end{abstract}

*Email: azzaroni@inifta.unlp.edu.ar

Homepage: http://softmatter.quimica.unlp.edu.ar 


\section{Introduction}

Environment responsive microgel particles have been the focal point of significant research activity in the past years due to their versatile applications in multiple technological fields. ${ }^{1}$ Microgel particles are formed by cross-linked polymer chains, which can incorporate different functional units. These microgels can be designed to undergo a volume phase transition in response to specific stimuli such as changes in temperature, salt concentration, $\mathrm{pH}$, or solvent quality. In particular, the study of microgels with temperature sensitivity near physiological conditions has drawn increasing attention due to potential applications in drug delivery, ${ }^{2}$ sensing, ${ }^{3,4}$ regenerative medicine, ${ }^{5}$ therapeutic medicine ${ }^{6}$ and chemical separation, ${ }^{7}$ to name a few examples.

The most studied thermosensitive polymer is poly( $N$-isopropylacrylamide) (PNIPAM), which exhibits a nominal lower critical solution temperature (LCST) in the range $30-34{ }^{\circ} \mathrm{C}$. Chemically cross-linked PNIPAM-based microgels in aqueous solution reveal a drastic particle size decrease upon heating above the LCST. This volume transition has been extensively studied by different experimental techniques, including static and dynamic light scattering, ${ }^{8,9,10,11,12,13,14,15}$ small angle neutron scattering (SANS), ${ }^{16,17}$ turbidimetry, ${ }^{12}$ atomic force microscopy ${ }^{18}$ and rheological measurements ${ }^{19}$ among other techniques.

The thermal response of PNIPAM can be summarized as follows: below the LCST, the solventpolymer attractions are stronger than the polymer-polymer ones, and consequently microgel particles are highly hydrated (swollen) in water; above the LCST, polymer-polymer interactions are stronger than polymer-solvent attractions, which leads to a more compact structure. ${ }^{20}$ Interestingly, the transition temperature can be changed by co-polymerization in the presence of other monomers, as is the case of PNIPAM-based microgels incorporating ionic groups. ${ }^{21,22,23,24,25,26,27}$ This control over the LCST is particularly relevant in view of potential applications of thermoresponsive ionic microgels as active transport agents and controlled drug delivery vehicles. ${ }^{28,29}$

Using PNIPAm-based microgels in the development of new thermally sensitive nanomaterials for the encapsulation and delivery charged drugs requires enhancing the microgel load capacity. This can be achieved through the incorporation of ionic groups into the PNIPAM network. In particular, incorporating ionizable co-monomers leads to PNIPAM-based microgels with additional response to changes in $\mathrm{pH}$ and salt concentration. In this regard, poly(N-isopropylacrylamide-co-methacrylic acid), P(NIPAM-co-MAA), particles represent an interesting model system of ionizable, thermoresponsive microgels increasingly employed in multiple practical applications, ${ }^{30},{ }^{31}$ including emulsion stabilization, ${ }^{32,33}$ layer-by-layer assembly, ${ }^{34}$ gelling, ${ }^{35}$ drug delivery, ${ }^{36}$ thin film ${ }^{34,37}$ and responsive materials preparation, ${ }^{38}$ microencapsulation, ${ }^{39}$ and nanomaterials templating, ${ }^{40,41}$ among others.

When considering the thermoresponsive properties of ionic PNIPAM-based microgels in the controlled design of new functional materials with technological applications, it is increasingly important to elucidate the molecular mechanisms that determine the microgels behavior. Rather than consisting of monolithic architectures, these nanostructures are confined arrays of different contrasting functions in which the delicate interplay between physical interactions and chemical equilibria can lead to unexpected properties. For example, it has been long considered that charged PNIPAM microgels experience strong electrostatic repulsive interactions leading to a rather incompressible behavior. However, this vision changed after Richtering and co-workers reported that charged microgels can be compressed more easily than neutral microgels; consequently, electrostatic repulsions do not solely determine microgel compressibility in solution. ${ }^{42,43}$ 
In the traditional picture of temperature-induced volume phase transitions in PNIPAM-based microgel solutions, a monotonic, relatively sharp, decrease in particle size occurs upon heating above the transition temperature. ${ }^{44,23}$ On the contrary, we have observed an unexpected nonmonotonic evolution of particle size when increasing the solution temperature. More precisely, we report that P(NIPAM-co-MAA) microgels reproducibly experience a marked swelling at temperature values close to but below the LCST. Thus, this swelling occurs in the temperature range where NIPAm is water-soluble. Further heating the solution leads to the shrunken state typically observed at temperatures above the LCST. Both the thermally induced swelling and the dimensions of the dispersed nanoobjects below the LCST are strongly dependent on the salt and microgel particle concentration.

The aim of this work is two-fold: to report, for the first time, the singular concentration-dependent thermo-active properties of ionizable PNIPAm microgel particles, and to elucidate the molecular origin of such unexpected and novel behavior. To meet these goals, we have combined dynamic light scattering (DLS) and nuclear magnetic resonance (NMR) studies with molecular-level theoretical calculations of the microgel system. Describing supramolecular organization inside microgels can be challenging due to the complexity of the experimental system. What molecular interactions drive the swelling of P(NIPAM-co-MAA) microgels below LCST? Both inter-microgel steric compressions and NIPAM-MAA hydrogen bond formation inside a microgel favor a more compact particle. ${ }^{45,46}$ At the $\mathrm{pH}$ of the experiments, the acid-base equilibrium dictates most MAA to be charged, which favors particle swelling due to intra-microgel electrostatic repulsions. Theoretical attention has been directed toward a better understanding of this interplay between physical interactions and chemical equilibriums that occurs at the nanometer length-scale and determines the unusual thermal-response of P(NIPAM-co-MAA) microgel particles, which precedes NIPAm collapse above its LCST.

\section{Results and Discussion}

Thermosensitive poly(N-isopropylacrylamide-co-methacrylic acid) (P(NIPAM-co-MAA)) microgel particles have been synthesized by free-radical precipitation co-polymerization and characterized in aqueous solutions by means of dynamic light scattering in the temperature range between 14 and 44 ${ }^{\circ} \mathrm{C}$ (see the Electronic Supporting Information file for further details). Figure 1 shows the variation of the hydrodynamic diameter, $D_{H}$, as a function of temperature when the microgel particles are exposed to different salt concentrations (at microgel concentration of $5 \mathrm{mg} / \mathrm{mL}$ ). At low temperature $\left(14{ }^{\circ} \mathrm{C}<\mathrm{T}<20^{\circ} \mathrm{C}\right)$, the particle diameter depends on the $\mathrm{KCl}$ concentration.

The incorporation of ionic or ionizable groups into the microgel polymer network strongly influences its response upon changes on the solution ionic strength. At the intermediate $\mathrm{pH}$ value used in this work $(\mathrm{pH} \sim 5)$, the acid groups stemming from methacrylic acid are expected to be partially deprotonated resulting in a negatively charged network, which was confirmed by zpotential measurements. With increasing ionic strength, the electrostatic repulsions between charged monomer units are more screened, resulting in partial deswelling of the particle. Figure 1 shows that P(NIPAM-co-MAA) microgel size changed from 800 to $620 \mathrm{~nm}$ when the concentration of $\mathrm{KCl}$ was varied from 5 to $50 \mathrm{mM}$. 


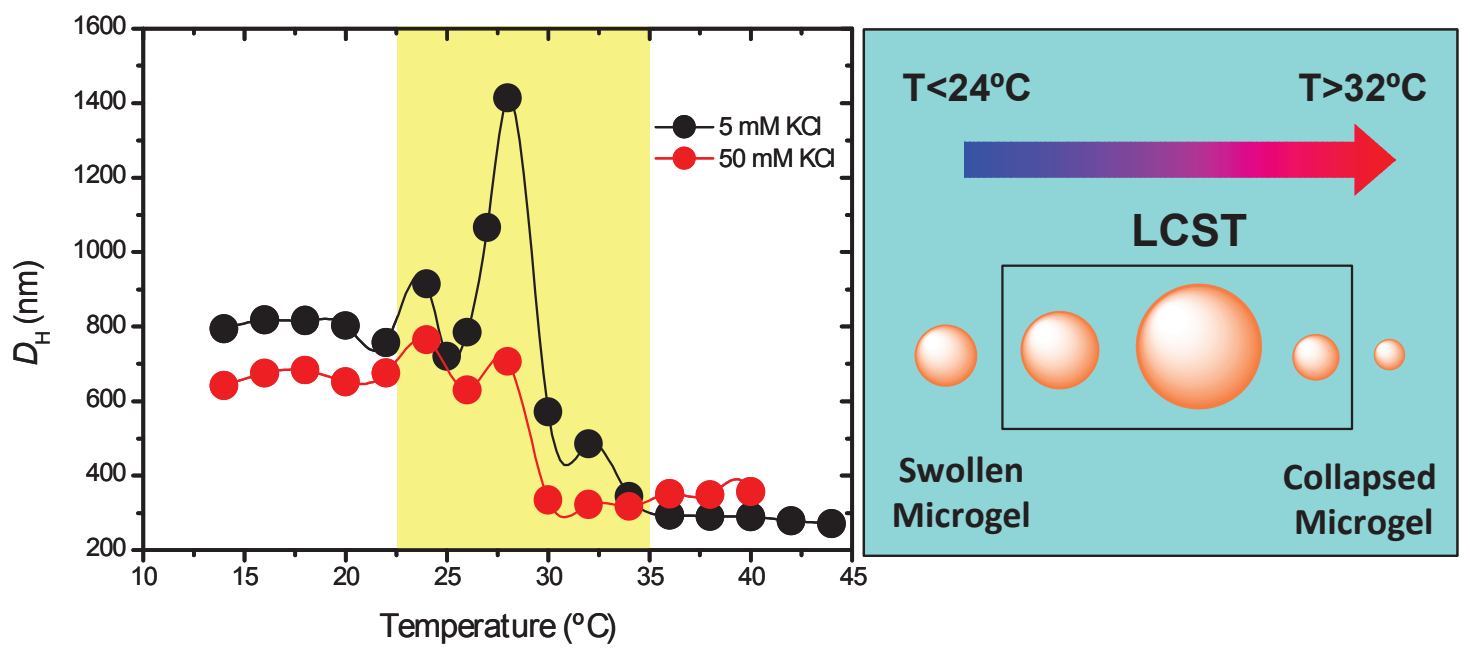

Figure 1. (Left) Swelling curves of P(NIPAM-co-MMA) microgels resulting from dynamic light scattering experiments. The hydrodynamic diameter $\left(D_{\mathrm{H}}\right)$ of the microgel in suspensions containing $5 \mathrm{mM}$ (black) and $50 \mathrm{mM} \mathrm{KCl}$ was determined as a function of temperature. (Right) Schematic depiction of the dimensional changes taking place in the microgel upon solution temperature increase.

Upon increasing temperature intriguing differences are observed between microgel particles dispersed in 5 and $50 \mathrm{mM} \mathrm{KCl}$. In the higher ionic strength conditions, the size-temperature profile shows the typically expected behavior. Namely, microgel particle diameter is a monotonic function of temperature. A sharp decrease in size occurs when the solution temperature is increased above the volume phase transition temperature (VPTT). At this point, the particles experience a transition from a relatively swollen configuration at lower temperatures to a collapsed state at higher temperatures. However, in stark contrast with the previous observation, the microgel particle exhibits a marked increase in size below the VPTT at low ionic strength conditions. To the best of our knowledge, only size decrease has been reported when solution temperature is increased for PNIPAM-based microgels. We argue that previous studies on the thermoactive properties P(NIPAM-co-MAA) microgel did not report this behavior because samples were measured under high ionic strength conditions. To conclude this paragraph, we mention that the microgel size above the VPTT is the same independently of the salt concentration.

The influence of ionic strength on the presence of this unusual behavior is a clear indication that electrostatic interactions may be responsible for this singular phenomenon. Though this is true, we will show that such behavior results from a complex interplay between the acid-base equilibrium of MAA units, the electrostatic interactions between dissociated MAA segments, and the formation of NIPAM-MAA hydrogen bonds. This delicate balance between these intra-microgel interactions can be critically altered by the steric compression resulting from the microgel concentration.

This unusual thermoactive behavior of P(NIPAM-co-MAA) microgel particles is drastically affected by the concentration of the microgel in the aqueous solution. Figure 2 shows measurements performed in low ionic strength conditions in the presence of different amounts of microgel. Interestingly, the hydrodynamic diameter measured at low temperatures $\left(14{ }^{\circ} \mathrm{C}<\mathrm{T}<20{ }^{\circ} \mathrm{C}\right)$ strongly depends on the microgel concentration. Increasing microgel concentration from 1 to 5 $\mathrm{mg} / \mathrm{mL}$ results in a diameter decrease from 1300 to $800 \mathrm{~nm}$. To understand this observation, we 
need to consider that in the case of ionic microgels two different phenomena can lead to shrinkage as microgel particle concentration increases: inter-microgel steric compression and ion-induced deswelling. Steric compression occurs when the particle concentration increases. That is, the volume fraction occupied by microgels increases, and individual particles reduce their size due to physical constraints imposed by surrounding microgel particles. On the other hand, ionic microgels may exhibit deswelling due to variations in the relative concentration of counterions inside and outside the polymeric particle. Typically, enough counterions are located inside the polymer network, where the charged monomers are confined, to neutralize the electric charge of the microgel. This is due to the entropy loss of confining ions inside the microgel. However, when the particle concentration increases, the free volume in the outer solution decreases, which modifies the entropic balance between internal and external ions with the concomitant variation in the osmotic pressure.

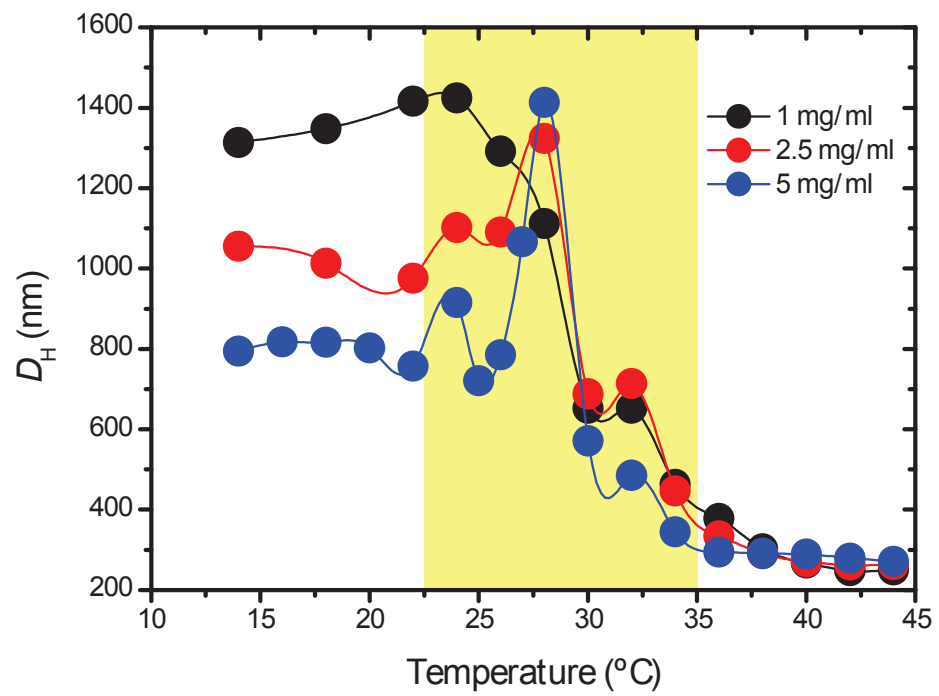

Figure 2. Swelling curves of P(NIPAm-co-MMA) microgels resulting from dynamic light scattering experiments. The hydrodynamic diameter $\left(D_{\mathrm{H}}\right)$ of the microgel in suspensions of containing different microgel concentrations, $1 \mathrm{mg} / \mathrm{mL}$ (black), $2.5 \mathrm{mg} / \mathrm{mL}$ (red) and $5 \mathrm{mg} / \mathrm{mL}$ (blue), was determined as a function of temperature in $5 \mathrm{mM} \mathrm{KCl}$.

The effects of both ion-induced deswelling and steric compression on particle shrinkage have been recently addressed by Romeo et al. ${ }^{45,46}$ These authors studied the reduction in size of ionic microgels based on poly( $\mathrm{N}$ isopropylacrylamide-co-acrylic acid) in response to increasing number of particles to show that particle shrinkage originates primarily from steric compression, and that the effect of ion-induced deswelling of the polymer network is negligible. Hence, we can ascribe the observed changes in particle size upon increasing the microgel concentration to increasing steric compression exerted by neighboring P(NIPAM-co-MAA) particles.

As aforementioned, microgel concentration strongly influences the thermoresponse, whereas the unusual thermoactive effect is more pronounced as we increase the microgel content in the solution. Indeed, dilute solutions $(1 \mathrm{mg} / \mathrm{mL})$ do not exhibit the thermo-induced microgel swelling. However, more concentrated solutions, 2.5 and $5 \mathrm{mg} / \mathrm{mL}$, exhibit a $D_{\mathrm{H}}$ increase from 1030 to $1340 \mathrm{~nm}$ and from 810 to $1430 \mathrm{~nm}$, respectively, when heating the microgel solution from 15 to $28{ }^{\circ} \mathrm{C}$. The critical role of microgel concentration indicates that inter-particle interactions - compression steric 
forces - play a critical role in defining the thermoresponsive properties of the microgel at temperatures below the VPTT. Above such temperature, the microgels collapse to the same particle size measured in experiments varying the salt concentration, $\sim 200 \mathrm{~nm}$ (see both Figs. 1 and 2).

The characteristics of the hydration layer plays an important role in the coil-to-globule transition of thermoresponsive polymers. ${ }^{47,48}$ To gain further insights into the molecular mechanisms governing the unusual thermal behavior, we have performed temperature-dependent NMR studies. The rationale behind these experiments is to investigate the nature of water molecules inside the microgel network. To this end we measured transverse relaxation times $\left(T_{2}\right)$ to obtain information about the dynamics of water molecules during the unexpected swelling transition. In principle, the Carr-Purcell-Meiboom-Gill (CPMG) decay can have several contributions that result from the polymeric matrix, the water in tight contact with it, and the bulk water molecules (see the Electronic Supporting Information file for further information and experimental details). The $T_{2}$ parameter corresponding to the polymer segments is extremely short and not observable under our experimental conditions (see ESI file). Under the measurement conditions, bulk water is the main contribution to the NMR signal. An inversion pulse was applied prior to the CPMG to suppress most of bulk signal, so as to obtain information from hydration layers.

In order to clarify the unusual thermal-response before collapse of our microgel particles, NMR experiments have been carried out in the temperature range between 17 and $31{ }^{\circ} \mathrm{C}$. Our NMR experiments revealed the presence of four different relaxation times in the low temperature regime (i.e., in the range of temperature below the microgel collapse due to NIPAm hydrophobicity). Figures 3 and 4 present the behavior of four different relaxation times with temperature in $\mathrm{P}(\mathrm{NIPAM}$-co-MAA) microgel at 1 and $5 \mathrm{mg} / \mathrm{mL}$ respectively, both $5 \mathrm{mM} \mathrm{KCl}$. We assign these relaxation times to bulk water $\left(T_{2}{ }^{\mathrm{I}} \sim 2 \mathrm{~s}\right)$, water inside microgel network $\left(T_{2}{ }^{\mathrm{II}} \sim 100 \mathrm{~ms}\right)$, and two other values corresponding to water molecules in the hydration layer of each of two different network monomers $\left(T_{2}{ }^{\mathrm{III}} \sim 10 \mathrm{~ms}\right.$ and $\left.T_{2}^{\mathrm{IV}} \sim 6 \mathrm{~ms}\right)$. These four populations of water molecules are labeled $W_{\mathrm{I}}, W_{\mathrm{II}}, W_{\mathrm{III}}$ and $W_{\mathrm{IV}}$, respectively. As expected, bulk water $T_{2}\left(W_{\mathrm{I}}\right)$ remains relatively constant when temperature increases (see Fig. 3 and 4). In addition, the relaxation time of water molecules inside the polymer network $\left(W_{\mathrm{II}}\right)$ is consistent with values for water molecules trapped in other polymeric systems. ${ }^{49,50,51}$

Several authors have studied solvation of NIPAM polymers and copolymers..$^{52,53,54,55}$ Typically, water molecules are divided in three categories: (a) free water, (b) water in the first hydration layer of the polymer and (c) the non-freezing water due to their thermal behavior. ${ }^{56,57,52,58}$ This classification is in agreement with the different water populations described in this work according to the $T_{2}$ values observed, where free water corresponds to $W_{\mathrm{I}}$, water in the first hydration layer of the polymer to $W_{I I I}$ and $W_{I V}$ and non-freezing water corresponds to $W_{\mathrm{II}}$. Considering this classification of water molecules embedded in the microgel surroundings our NMR experiments indicate that, in principle, at low polymer concentrations and low temperature conditions both types of monomers in the polymer network are in contact with the solvent ( $W_{\text {III }}$ and $W_{\text {IV }}$ ) (see Fig. 3 ). Upon increasing temperature, the PNIPAM chains suffer a coil-to-globule transition and consequently $T_{2}$ values vary consistently. At temperatures above $30^{\circ} \mathrm{C}$, the polymeric segments are in a globular state and the water distribution presents only a subtle variation showing that even in the collapsed state the polymeric network has water molecules in its inner environment. This observation is in agreement with theoretical calculations reported for similar matrixes. ${ }^{59,60,61,62}$ 


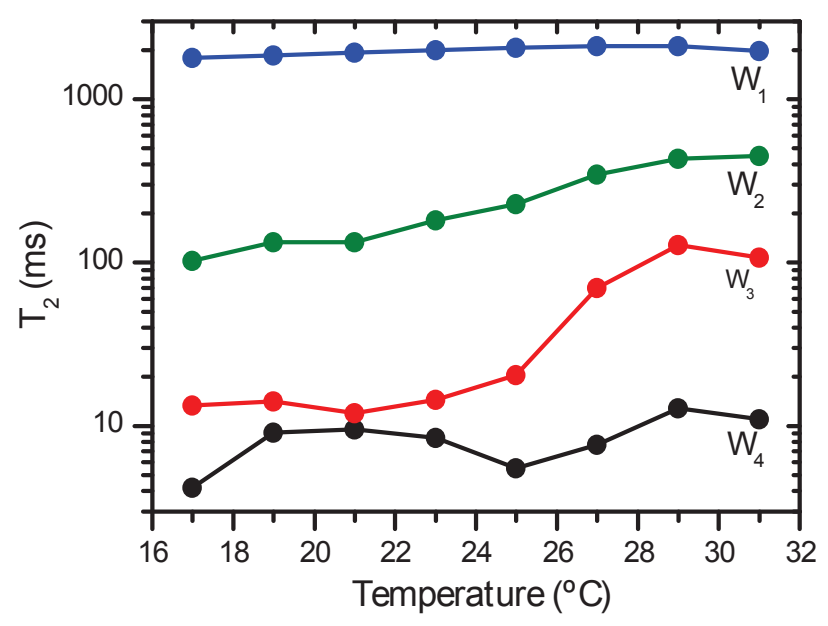

Figure 3. Relaxation times $\left(\mathrm{T}_{2}\right)$ for water molecules contained in the P(NIPAm-co-MAA) microgel environment (at $1 \mathrm{mg} / \mathrm{mL}$ ) as a function of temperature.

As shown Fig. 4, at higher microgel particle concentrations four different $T_{2}$ values were also determined and assigned to aforementioned water categories. However, in contrast with the observations at low microgel particle concentration, the $W_{\text {IV }}$ population only appears at temperatures above $25{ }^{\circ} \mathrm{C}$. This interesting observation suggests that at lower temperatures, there is little water (under the detection limit) in the first hydration layer of one of the monomer units. These results are concomitant with experiments at high microgel particle concentration $(5 \mathrm{mg} / \mathrm{mL})$ that indicate physicochemical and interesting hydration changes in the temperature domain, that correspond to the unexpected swelling of the microgel at $25{ }^{\circ} \mathrm{C}$, which precedes the collapse occurring at higher temperatures (see Fig. 2).

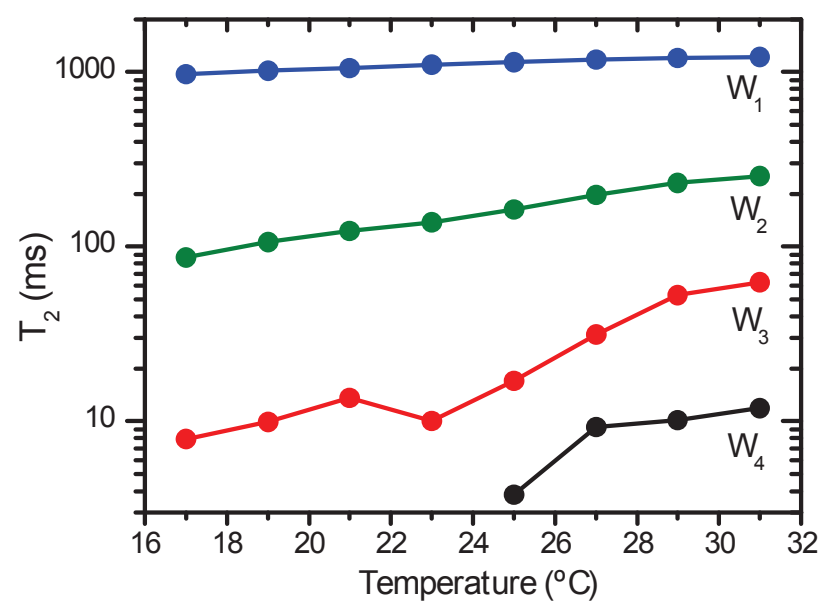

Figure 4. Relaxation times $\left(\mathrm{T}_{2}\right)$ for water molecules contained in the P(NIPAM-co-MAA) microgel environment (at $5 \mathrm{mg} / \mathrm{mL}$ ) as a function of temperature.

As discussed above, at low temperatures an increase in microgel particle concentrations leads to a decrease in particle size. ${ }^{59}$ Under these conditions, in the interior of a microgel NIPAM and MAA units can form hydrogen bonds. Sukhishvili and co-workers have indicated that hydrogen bonding 
between MAA and NIPAM units occurs in macromolecular systems containing both types of monomers. ${ }^{60}$ Based on the formation of NIPAM-MAA hydrogen bonds and on the molar composition of the microgels studied in this work (0.65:0.35 NIPAM:MMA) (see ESI file), we assign $W_{\text {III }}$ and $W_{\text {IV }}$ to the first coordination sphere of NIPAM and MAA, respectively.

If a large fraction of MAA monomers are H-bonded with NIPAM units, water molecules are depleted from the first coordination sphere of former network component. This explains why $\mathrm{W}_{\mathrm{IV}}$ relaxation time is not observed in our NMR experiments at high microgel concentrations and low temperature conditions (see schematic representation in Fig. 5). Hydrogen bonds break when the temperature increases above that of swelling, $\sim 25^{\circ} \mathrm{C}$, and the presence of water molecules in the solvation layer of now free MAA units results in the appearance of $\mathrm{W}_{4}$ relaxation time. On the other hand, the microgel architecture is richer in NIPAM. Even if a high degree of NIPAM-MAA binding occurs, a large fraction of NIPAM units remains unbound. Thus, this allows for a detectable amount of water molecules in the first coordination sphere of NIPAM, resulting in the presence of the $\mathrm{W}_{\text {III }}$ relaxation time in all the temperature range and microgel concentrations considered in the NMR experiments.

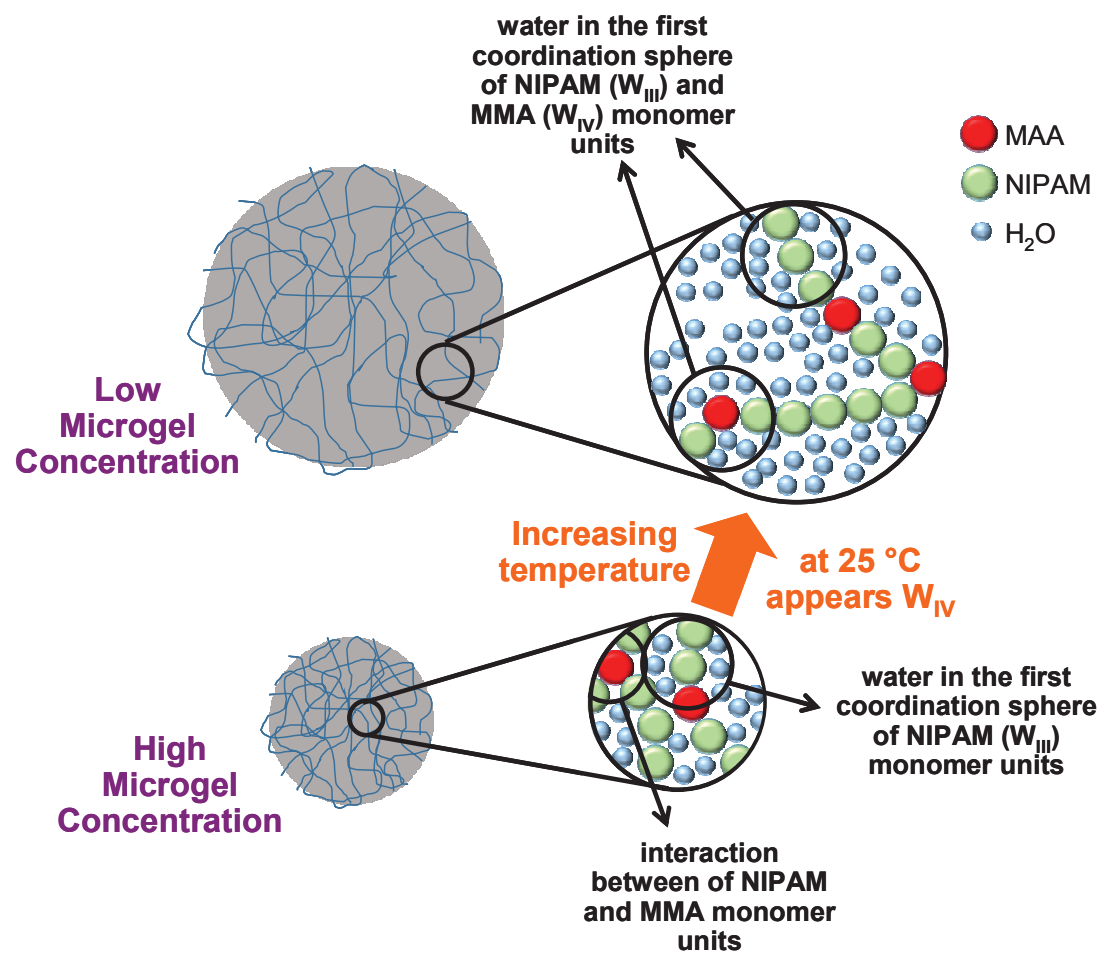

Figure 5. Schematic depiction of the processes taking place within the microgel environment upon increasing temperature and microgel concentration.

We have performed theoretical calculations for intra-network binding and charge regulation in P(NIPAM-co-MAA) microgels. We have considered a polymer network formed by cross-linked random copolymer chains. This network contains two types of segments, NIPAM and MAA units (see Fig. 6). The theoretical approach we employ explicitly describes MAA charge regulation and the formation of MAA-NIPAM complexes. Both the fraction of charged MAA units and the degree of complex formation are determined by the interplay between the several physicochemical terms 
that contribute to the free energy. The main idea behind this method is to write the thermodynamic potential as a function of the probability of each spatial conformation of the network, and include all relevant repulsive and attractive interactions, in addition to the different chemical states of the different network components. For a MAA unit these chemical states are charged, protonated and bound, while a NIPAM unit can either be free or bound. Optimization of the thermodynamic potential provides the probability of each network conformation and the different chemical states. Thus, this theory does not assume any particular state of charge and binding, rather they result from a calculation for each set of experimentally controlled variables.

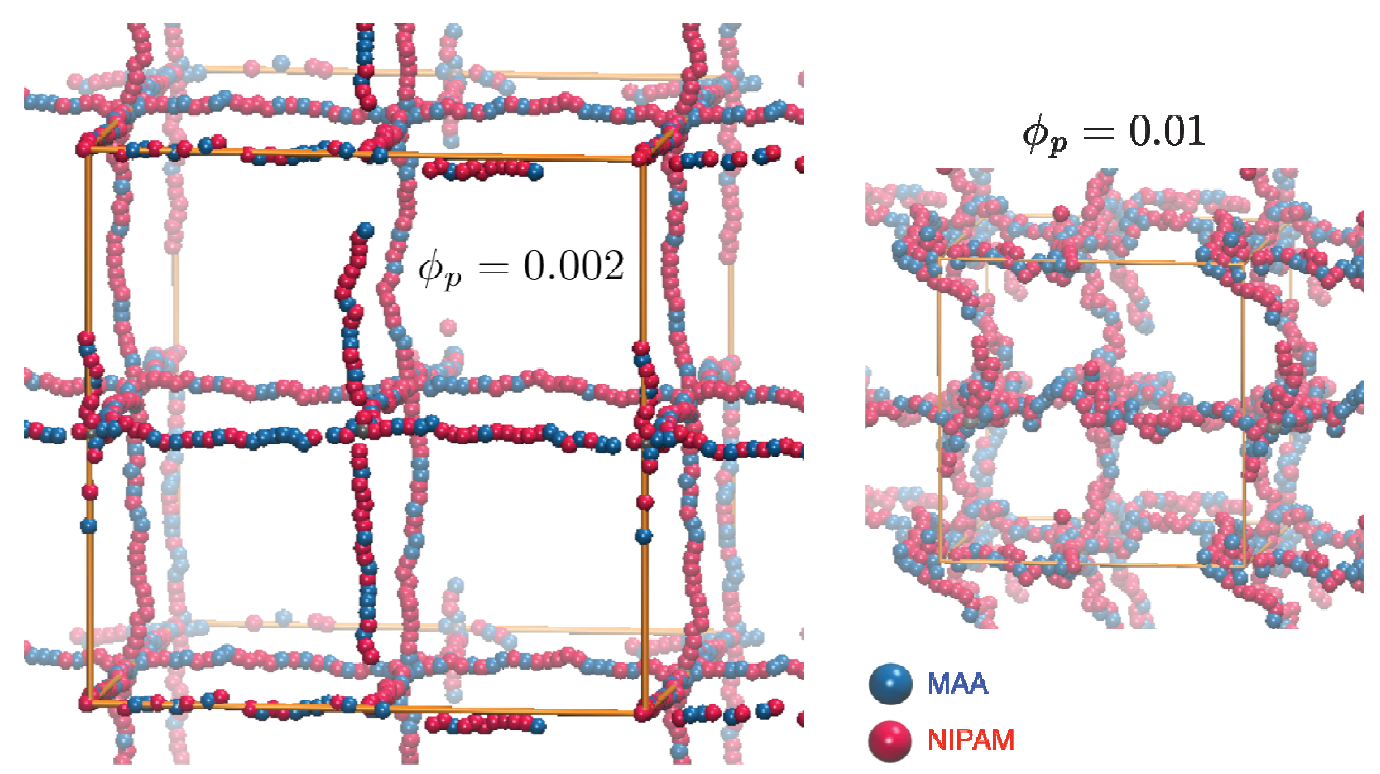

Figure 6. The scheme represents the molecular model of polymer network used in our theoretical calculations. Random NIPAM-co-MAA copolymer chains are crosslinked at nodal units. Each crosslink joins six 25 segment-long chains; the polymer segment length is $0.5 \mathrm{~nm}$. The two panels correspond to different volume fractions of polymer inside a single microgel, $\phi_{p}$. These volume fractions represent the relatively compact $\left(\phi_{\mathrm{p}}=0.01\right)$ and swollen $\left(\phi_{\mathrm{p}}=0.002\right)$ microgels observed in Fig. 2 for $5 \mathrm{mM}$ salt solutions and at temperatures lower than the VPTT. Orange tubes enclose the calculation box; full-3D periodic boundary conditions are applied in the calculations.

The set of different conformations of microgel network is an input of this theory. Thus, in order to calculate results, we need to define a molecular model for the P(NIPAm-co-MAA) network. The polymer network is composed of crosslinked 25-segment long polymer chains; the segment length is $l_{\text {seg }}=0.5 \mathrm{~nm}$. Each segment is randomly labeled as either a MAA or a NIPAM unit, the only constraint being that the MAA:NIPAM ratio be 0.35:0.65 as determined in our experimental systems. The different conformations of the network are obtained using Molecular Dynamics simulations (see Supporting Information).

Figure 7A shows the fraction of MAA units of the network that are bound to NIPAM segments, $<x_{\text {bnd }}>$, as a function of the standard binding free energy of the isolated pair, $\Delta G^{\circ}$, under different conditions. Similarly, panel B shows the fraction of dissociated or charged MAA units, $\left\langle x_{\mathrm{dis}}>\right.$, under the same conditions. The rationale behind these calculations is that an increase of temperature in the range of unusual behavior $\left(24-28{ }^{\circ} \mathrm{C}\right)$ can be effectively represented by a decrease in the binding affinity between MAA and NIPAM. The x-axis in both panels of Fig. 7 covers the typical 
range of standard free energies of hydrogen bonding. The two different polymer volume fractions considered in Fig. $7\left(\phi_{p}=0.01\right.$ and 0.002) represent the relatively shrunken/compressed and swollen states of the microgels observed in Fig. 2 at 24 and $28{ }^{\circ} \mathrm{C}$, respectively, for $5 \mathrm{mM}$ salt solutions. The ratio between these volume fractions is equivalent to that observed in our experimental microgels.
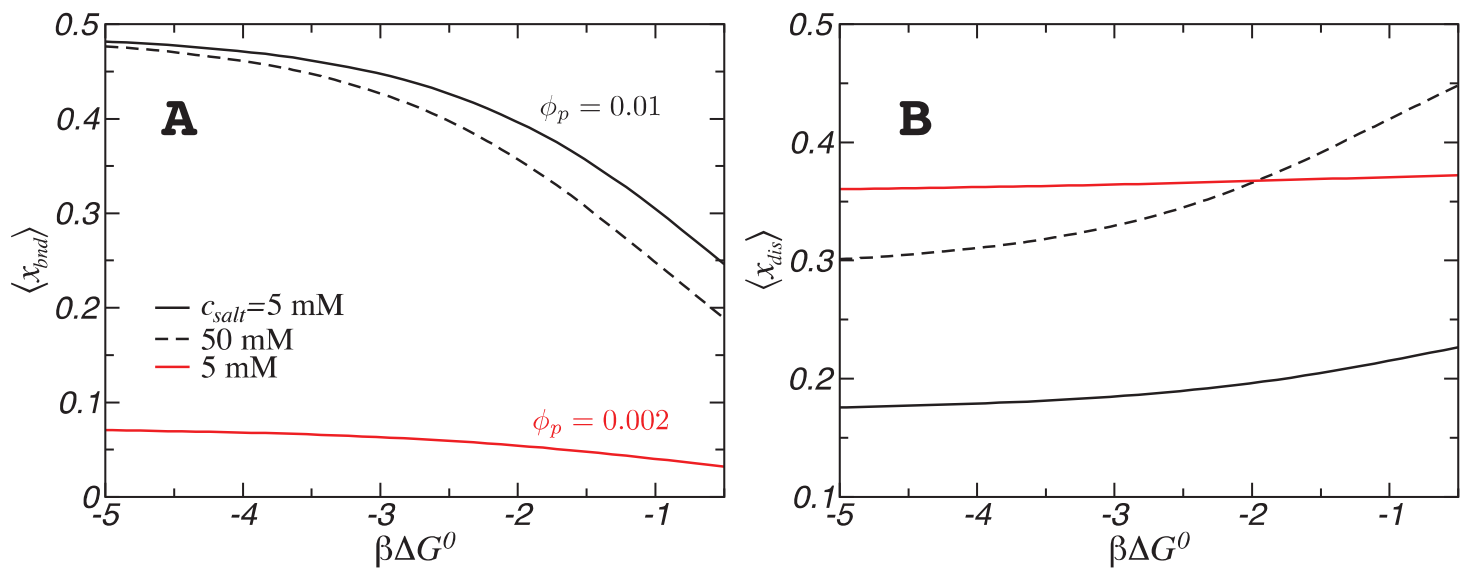

Figure 7. The panels show (A) the faction of MAA units that are bound to NIPAM, $<x_{\text {bnd }}>$, (A) and the fraction of acid units that are dissociated (B) as a function of the MAA-NIPAM standard binding free energy (in units of room temperature thermal energy). The solution $\mathrm{pH}$ is 5 in all cases, while two salt concentrations are considered, 5 and $50 \mathrm{mM}$ (solid and dashed lines, respectively). Results for a relatively compressed network $\left(\phi_{\mathrm{p}}=0.01\right.$; black curves $)$ and a swollen network $\left(\phi_{\mathrm{p}}=\right.$ 0.002 ; red curve) are presented.

The intrinsic $\mathrm{pKa}$ of MAA is 4.65. From ideal solution considerations, we a priori expect more than $50 \%$ of MAA units to be dissociated at $\mathrm{pH} 5$. However, only $15-25 \%$ of these units are charged for $\phi_{\mathrm{p}}=0.01$ (solid-black curve in Fig. 7B). The reason for this low dissociation is the confinement of charged groups. A higher degree of dissociation would lead to strong electrostatic repulsions between localized MAA negative charges in the compressed network. So, due to the relatively high volume fraction, the network dissociates less than expected from ideal solution considerations in order to reduce intra-network electrostatic repulsions.

Less dissociation, however, pays the price of increasing the chemical free energy that describes the acid-base equilibrium, which is minimized when the dissociation is precisely that of the ideal solution. In the absence of an external force, the compressed volume fraction is not thermodynamically stable, since the $\phi_{\mathrm{p}}=0.002$ network has a lower free energy. Swelling allows for higher dissociation (see red curve in Fig. 7B), which relaxes the acid-base equilibrium frustration, while at the same time increasing the effective distance between like-charged network segments.

Therefore, in the experiments the higher volume fraction (smaller microgel size) observed at $\mathrm{T}<24$ ${ }^{\circ} \mathrm{C}$ results from the combination of an external force introduced by inter-microgel steric compressions, and the formation of a high degree of MAA-NIPAM complexes. However, as the strength of the interaction decreases with temperature, complexes are less likely to form and the stabilizing intra-network force significantly weakens. Thus, the gel swells to increase the degree of dissociation. This unusual behavior is not experimentally observed when the salt concentration is 50 $\mathrm{mM}$. At this salt concentration, our theoretical calculations show that the degree of dissociation in 
the compressed network is close to that of the ideal solution, even if the strength of the interaction and the degree of binding are both high (see dashed lines in Fig. 7). Thus, dissociation in the polymer is nearly ideal, which results in no driving force for swelling under these conditions as $\Delta G^{0}$ decreases.

\section{CONCLUSIONS}

Solutions of PNIPAM-based microgels experience a monotonic, relatively sharp, decrease in particle size when the solution is heated above the volume phase transition temperature. ${ }^{44,23} \mathrm{We}$ have observed this traditional behavior with DLS for P(NIPAM-co-MAA) microgel solutions at low particle concentration and high $\mathrm{KCl}$ concentration. However, at higher microgel densities and lower salt concentrations, we report for the first time an unexpected non-monotonic evolution of particle size when increasing the microgel solution temperature. Under such conditions, our DLS experiments show that microgels reproducibly experience a marked swelling at temperature values where NIPAm is water-soluble (i.e., below the VPPT). Further heating the microgel solution leads to the shrunken state typically observed at temperatures above the VPTT.

Our NMR studies reveled that one of the co-monomers (MAA) is significantly hydrated only after the unexpected swelling. This observation together with previous studies ${ }^{60}$ led us to the conclusion that MAA-NIPAM complexes occur inside the compressed microgel observed at low temperature. These complexes break when the temperature increases and the microgel swells, resulting in the hydration of the now free MAA monomer and the appearance of its NMR signal.

To complete the picture of the molecular origin of this unusual swelling, we have performed theoretical calculations. At low temperature $\left(<25^{\circ} \mathrm{C}\right)$, microgel particles in concentrated solutions (microgel concentration $>2.5 \mathrm{mg} / \mathrm{ml}$ ) having low salt concentration $(5 \mathrm{mM})$ are compressed due to inter-particle steric compression. Our theoretical results show that the formation of MAA-NIPAM complexes can help stabilize a compressed microgel at low temperature. Stability of compressed microgels, however, comes at the expense of low MAA dissociation at a $\mathrm{pH} \mathrm{5,} \mathrm{where} \mathrm{most} \mathrm{of} \mathrm{the}$ acid groups are expected to be deprotonated from ideal (dilute) solution considerations. This low degree of charge establishes inside the microgels in order to reduce the intra-network electrostatic repulsions between the highly confined acid groups in the compressed network. The price paid for reducing the electrostatic energy is increasing the chemical free energy of the acid-base equilibrium, which is minimum when the dissociation is that of the dilute solution.

As temperature increases, and the effective strength of the binding interaction between MAA and NIPAM units decreases (in absolute value), MAA-NIPAM complexes are much less likely to occur. Thus, the stabilizing contribution of the binding free energy to the compressed microgels weakens. Then, the microgel swells to increase the degree of MAA dissociation and relax the chemical free energy of the acid-base equilibrium, while at the same time increasing the effective distance between charged segments. At higher salt concentrations MAA units are significantly dissociated even in the compressed microgel. Thus, the driving force for swelling (i.e., the constraint of the acid-base equilibrium to increase charge, which induces repulsions between charged segments) is not present.

We consider that this unusual physicochemical properties exhibited by ionizable PNIPAM-based microgels might have strong implications for the use and applications of thermoresponsive microgels in multiple fields, and might be especially important in the molecular design of polymerbased vehicles for drug delivery. 


\section{ACKNOWLEDGMENTS}

This work was supported by CONICET, ANPCyT (PICT-2010-2554, PICT-2013-0905), Fundación Petruzza and the Austrian Institute of Technology GmbH (AIT-CONICET Partner Group: "Exploratory Research for Advanced Technologies in Supramolecular Materials Science" - Exp. 4947/11, Res. No. 3911, 28-12-2011). J.M.G., G.S.L., R.H.A. and O.A are staff members of CONICET.

\section{REFERENCES}

1 J. B. Thorne, G. J. Vine and M. J. Snowden, Colloid Polym. Sci., 2011, 289, 625.

2 J. Ramos, A. Imaz, J. Callejas-Fernández, L. Barbosa-Barros, J. Estelrich, M. Quesada-Pérez and J. Forcada, Soft Matter, 2011, 7, 5067.

3 R. A. Álvarez-Puebla, R. Contreras-Cáceres, I. Pastoriza-Santos, J. Pérez-Juste and L. M. Liz-Marzán, Angew. Chemie - Int. Ed., 2009, 48, 138.

4 K. C. C. Johnson, F. Mendez and M. J. Serpe, Anal. Chim. Acta, 2012, 739, 83.

5 B. R. Saunders, N. Laajam, E. Daly, S. Teow, X. Hu and R. Stepto, Adv. Colloid Interface Sci., 2009, 147-148, 251.

6 M. K. Nguyen and E. Alsberg, Prog. Polym. Sci., 2014, 39, 1235.

7 H. Kaşgöz, S. Özgümüš and M. Orbay, Polymer (Guildf)., 2003, 44, 1785.

8 J. Wu, B. Zhou and Z. Hu, Phys. Rev. Lett., 2003, 90, 048304.

9 T. Eckert and W. Richtering, J. Chem. Phys., 2008, 129, 124902.

10 A. M. Howe, S. Desrousseaux, L. S. Lunel, J. Tavacoli, H. N. Yow and A. F. Routh, $A d v$. Colloid Interface Sci., 2009, 147-148, 124.

11 B. H. Tan, R. H. Pelton and K. C. Tam, Polymer (Guildf)., 2010, 51, 3238.

12 N. C. Woodward, B. Z. Chowdhry, M. J. Snowden, S. a. Leharne, P. C. Griffiths and A. L. Winnington, Langmuir, 2003, 19, 3202.

13 A. N. S. John, V. Breedveld and L. A. Lyon, Bioengineering, 2007, 7796.

14 V. Wintgens and C. Amiel, Macromol. Chem. Phys., 2008, 209, 1553.

15 I. Varga, T. Gilányi, R. Mészáros, G. Filipcsei and M. Zrínyi, J. Phys. Chem. B, 2001, 105, 9071.

16 L. Arleth, X. Xia, R. P. Hjelm, J. Wu and H. U. Zhibinc, J. Polym. Sci. Part B Polym. Phys., $2005,43,849$.

17 B. R. Saunders, Langmuir, 2004, 20, 3925. 
18 S. M. Hashmi and E. R. Dufresne, Soft Matter, 2009, 5, 3682.

19 F. Scheffold, P. Díaz-Leyva, M. Reufer, N. Ben Braham, I. Lynch and J. L. Harden, Phys. Rev. Lett., 2010, 104, 1.

20 H. Senff and W. Richtering, J. Chem. Phys., 1999, 111, 1705.

21 K. Tauer, D. Gau, S. Schulze, A. Völkel and R. Dimova, Colloid Polym. Sci., 2009, 287, 299.

22 R. H. Pelton and P. Chibante, Colloids and Surfaces, 1986, 20, 247.

23 M. Karg, I. Pastoriza-Santos, B. Rodriguez-González, R. Von Klitzing, S. Wellert and T. Hellweg, Langmuir, 2008, 24, 6300.

24 K. Kratz, T. Hellweg and W. Eimer, Colloids Surfaces A Physicochem. Eng. Asp., 2000, 170, 137.

25 J. Kleinen and W. Richtering, Macromolecules, 2008, 41, 1785.

26 J. E. Wong and W. Richtering, Curr. Opin. Colloid Interface Sci., 2008, 13, 403.

27 E. Costa, M. M. Lloyd, C. Chopko, A. Aguiar-Ricardo and P. T. Hammond, Langmuir, 2012, 28, 10082 .

28 M. Malmsten, H. Bysell and P. Hansson, Curr. Opin. Colloid Interface Sci., 2010, 15, 435.

29 N. M. B. Smeets and T. Hoare, J. Polym. Sci. Part A Polym. Chem., 2013, 51, 3027.

30 M. Das, H. Zhang and E. Kumacheva, Annu. Rev. Mater. Res., 2006, 36, 117.

31 T. Hellweg, J. Polym. Sci. Part B Polym. Phys., 2013, 51, 1073.

32 S. Schmidt, T. Liu, S. Rütten, K. H. Phan, M. Möller and W. Richtering, Langmuir, 2011, 27, 9801.

33 B. Brugger and W. Richtering, Langmuir, 2008, 24, 7769.

34 M. J. Serpe, C. D. Jones and L. A. Lyon, Langmuir, 2003, 19, 8759.

35 W. Xiong, X. Gao, Y. Zhao, H. Xu and X. Yang, Colloids Surfaces B Biointerfaces, 2011, 84, 103.

36 M. J. Serpe, K. A. Yarmey, C. M. Nolan and L. A. Lyon, Biomacromolecules, 2005, 6, 408.

37 L. A. Lyon, Z. Meng, N. Singh, C. D. Sorrell and A. St John, Chem. Soc. Rev., 2009, 38, 865.

38 M. W. Spears, E. S. Herman, J. C. Gaulding and L. A. Lyon, Langmuir, 2014, 30, 6314.

39 S. Seiffert, J. Polym. Sci. Part A Polym. Chem., 2014, 52, 435.

40 J. E. Wong, A. M. Díez-Pascual and W. Richtering, Macromolecules, 2009, 42, 1229. 
41 A. P. H. Gelissen, A. J. Schmid, F. a. Plamper, D. V. Pergushov and W. Richtering, Polym. (United Kingdom), 2014, 55, 1991.

42 B. Brugger, S. Rütten, K. H. Phan, M. Moller and W. Richtering, Angew. Chemie - Int. Ed., 2009, 48, 3978.

43 K. Geisel, L. Isa and W. Richtering, Angew. Chemie - Int. Ed., 2014, 53, 4905.

44 T. Hoare and R. Pelton, Langmuir, 2004, 20, 2123.

45 G. Romeo, L. Imperiali, J. W. Kim, A. Fernández-Nieves and D. A. Weitz, J. Chem. Phys., 2012, 136, 124905.

46 G. Romeo and M. P. Ciamarra, Soft Matter, 2013, 9, 5401.

47 S. A. Deshmukh, G. Kamath, K. J. Suthar, D. C. Mancini and S. K. R. S. Sankaranarayanan, Soft Matter, 2014, 10, 1462.

48 X. Xu, G. Shan, Y. Shang and P. Pan, J. Appl. Polym. Sci., 2014, 131, 40589.

49 P. Y. Ghi, D. J. T. Hill and A. K. Whittaker, Biomacromolecules, 2002, 3, 991.

50 E. V. Silletta, M. I. Velasco, C. G. Gómez, R. H. Acosta, M. C. Strumia and G. A. Monti, Langmuir, 2014, 30, 4129.

51 S. Ghoshal, C. Mattea and S. Stapf, Chem. Phys. Lett., 2010, 485, 343.

52 T. Maeda, K. Yamamoto and T. Aoyagi, J. Colloid Interface Sci., 2006, 302, 467.

53 C. Hashimoto, A. Nagamoto, T. Maruyama, N. Kariyama, Y. Irisa, A. Ikehata and Y. Ozaki, Macromolecules, 2013, 46, 1041.

54 J. Chen, J. Spěváček and L. Hanyková, Macromol. Symp., 2014, 339, 24.

55 K. Shiraga, H. Naito, T. Suzuki, N. Kondo and Y. Ogawa, J. Phys. Chem. B, 2015, 119, 5576.

56 H. Hatakeyama and T. Hatakeyama, Thermochim. Acta, 1998, 308, 3.

57 P. S. Belton, Int. J. Biol. Macromol., 1997, 21, 81.

58 Z. H. Ping, Q. T. Nguyen, S. M. Chen, J. Q. Zhou and Y. D. Ding, Polymer, 2001, 42, 8461.

59 A. N. St. John, V. Breedveld and L. A. Lyon, J. Phys. Chem. B, 2007, 111, 7796.

60 Y. Lu, A. Zhuk, L. Xu, X. Liang, E. Kharlampieva and S. A. Sukhishvili, Soft Matter, 2013, 9, 5464 .

61 H. Muta, S. Kawauchi and M. Satoh, Colloid Polym. Sci., 2003, 282, 149.

62 Y. Maeda, M. Tsubota and I. Ikeda, Colloid Polym. Sci., 2003, 281, 79. 